\title{
UK Prospective Diabetes Study (UKPDS) 14: association of angiotensin-converting enzyme insertion/deletion polymorphism with myocardial infarction in NIDDM
}

\author{
B. D. Keavney ${ }^{1}$, C.R.K.Dudley ${ }^{1}$, I.M. Stratton ${ }^{2}$, R. R.Holman ${ }^{2}$, D.R. Matthews ${ }^{2}$, P. J. Ratcliffe ${ }^{1}$, R.C. Turner ${ }^{2}$ \\ ${ }^{1}$ Nuffield Department of Medicine, John Radcliffe Hospital, Headington, Oxford, UK \\ ${ }^{2}$ Diabetes Research Laboratories, Radcliffe Infirmary, Woodstock Road, Oxford, UK
}

Summary The deletion allele of the insertion/deletion polymorphism of the angiotensin-converting enzyme gene has been suggested to be an independent risk factor for myocardial infarction, particularly in subjects judged to be "low-risk" by the criteria of lipid status and body mass index. In a prospective, matched case-control study, we have investigated the role of this polymorphism as a risk factor for myocardial infarction in 173 newly-diagnosed British Caucasian non-insulin-dependent diabetic subjects taken from the United Kingdom Prospective Diabetes Study who subsequently developed myocardial infarction and 297 control subjects from the same study population matched for known cardiovascular risk factors including age at diagnosis of diabetes, gender, blood pressure, low-density lipoprotein cholesterol, high-density lipoprotein cholesterol, triglyceride and smoking habit. A trend towards increased risk conferred by homozygosity for the deletion al- lele was observed in cases (odds ratio 1.63, $p=0.09$ ). When the population was stratified according to the matched risk factors, the deletion allele was associated with myocardial infarction in those with low plasma low-density lipoprotein cholesterol (odds ratio $3.67, p=0.002$ ), or low triglyceride (odds ratio $3.14, p=0.005$ ). The strongest association of the deletion allele with myocardial infarction was observed in subjects with both low low-density lipoprotein cholesterol and low triglyceride levels (odds ratio 9.0, $p<0.001$ ). These results show that the deletion allele is a risk factor for myocardial infarction in non-insulin-dependent diabetic patients who have a favourable lipid profile. [Diabetologia (1995) 38: 948-952]

Key words Non-insulin-dependent diabetes mellitus, myocardial infarction, angiotensin-converting enzyme, genetics, risk factors.
Since the renin-angiotensin system plays a major role in vascular function, the angiotensin-converting enzyme (ACE) gene has been investigated as a candidate gene for susceptibility to vascular disease in humans. An insertion/deletion (I/D) polymorphism, ow-

Received: 11 October 1994 and in revised form: 31 January 1995

Corresponding author: Dr. B. Keavney, Wellcome Centre for Human Genetics, Windmill Road, Headington, Oxford OX3 $7 \mathrm{BN}$, UK

Abbreviations: ACE, angiotensin-converting enzyme; MI, myocardial infarction; I/D polymorphism, insertion/deletion polymorphism; I allele, insertion allele; D allele, deletion allele; DD genotype, homozygosity for the deletion allele; UKPDS, United Kingdom Prospective Diabetes Study. ing to the presence or absence of a 287 base pair fragment in intron 16 of the gene, was found to be in strong linkage disequilibrium with a major gene polymorphism, as yet uncharacterised, accounting for approximately $50 \%$ of the total variability in plasma ACE level [1-3]. A large case-control study carried out in France and Northern Ireland reported an increased risk of myocardial infarction (MI) in subjects with the DD genotype; subgroup analysis demonstrated this association to be confined to subjects classified as "low risk", based on a low body mass index (BMI) and low apolipoprotein B level [4]. In the control group of the same study population, the presence of the D allele was shown to be associated with an increased frequency of parental MI [5]. The D allele has been found to be more frequent in autopsied 
Table 1. Description of cases of myocardial infarction and matched control subjects

\begin{tabular}{|c|c|c|c|c|}
\hline & \multicolumn{2}{|c|}{ Matched excluding hypertensive status } & \multicolumn{2}{|c|}{ Matched including hypertensive status } \\
\hline & Cases & Control subjects & Cases & Control subjects \\
\hline$n$ & 163 & 163 & 134 & 134 \\
\hline Male gender $n(\%)$ & $112(69)$ & $112(69)$ & $96(72)$ & $96(72)$ \\
\hline Age (years) & $56.3(6.7)$ & $56.7(6.5)$ & $56.2(7.2)$ & $55.8(6.9)$ \\
\hline $\mathrm{BMI}\left(\mathrm{kg} / \mathrm{m}^{2}\right)$ & $29.1(5.5)$ & $28.8(4.6)$ & $29.0(5.5)$ & $28.9(4.6)$ \\
\hline $\mathrm{HbA}_{1 \mathrm{c}}(\%)$ & $9.3(8.0-10.5)$ & $8.8(7.5-10.8)$ & $9.3(8.1-10.5)$ & $8.7(7.2-10.7)^{\mathrm{a}}$ \\
\hline LDLC (mmol/1) & $3.64(3.05-4.45)$ & $3.61(3.05-4.38)$ & $3.62(3.05-4.31)$ & $3.64(3.04-4.32)$ \\
\hline $\mathrm{TG}(\mathrm{mmol} / \mathrm{l})$ & $1.68(1.31-2.52)$ & $1.63(1.67-2.42)$ & $1.58(1.31-2.31)$ & $1.60(1.28-2.24)$ \\
\hline HDLC $(\mathrm{mmol} / \mathrm{l})$ & $0.99(0.83-1.11)$ & $0.96(0.85-1.11)$ & $1.00(0.84-1.11)$ & $0.95(0.85-1.10)$ \\
\hline $\mathrm{SBP}(\mathrm{mm} \mathrm{Hg})$ & $139(126-150)$ & $140(123-152)$ & $138(125-150)$ & $135(123-149)$ \\
\hline $\mathrm{DBP}(\mathrm{mm} \mathrm{Hg})$ & $85(80-90)$ & $81(75-90)^{\mathrm{b}}$ & $85(78-90)$ & $81(77-89)$ \\
\hline HTNRx $n(\%)$ & $39(24)$ & $28(17)^{\mathrm{c}}$ & $24(18)$ & $24(18)$ \\
\hline Smoking $(\mathrm{n} / \mathrm{x} / \mathrm{c})$ & $29 / 72 / 62$ & $29 / 72 / 62$ & $26 / 56 / 52$ & $26 / 56 / 52$ \\
\hline
\end{tabular}

LDLC, LDL-cholesterol; TG, triglyceride; HDLC, high density lipoprotein cholesterol; SBP, systolic blood pressure; DBP, diastolic blood pressure; HTNRx, antihypertensive therapy; Smoking graded as never/ex/current

cases of definite or possible MI than in control subjects taken from a Belfast population [6]; a recent retrospective case-control study carried out in French subjects with non-insulin-dependent diabetes (NIDDM) also found association between the D allele and coronary heart disease (CHD) [7]. However, in a case-control study carried out in a Norwegian population, Bohn and colleagues [8] did not observe an association of DD genotype with MI, even in "low-risk" patients.

The UK Prospective Diabetes Study (UKPDS) is a multi-centre, prospective, randomised, intervention trial of 5102 newly-diagnosed patients with NIDDM which aims to determine whether improved blood glucose control will prevent complications and reduce the associated morbidity and mortality. A description of the study design has been published [9]. We have investigated the role of the I/D polymorphism of the ACE gene as a risk factor for MI in this high-risk population, in a case-control study, matching other cardiovascular risk factors as determined at entry to the study.

\section{Subjects, materials and methods}

One hundred and seventy-three patients who had suffered fatal or non-fatal MI since enrolment in UKPDS were ascertained (cases). Control subjects were patients drawn from UKPDS who had no evidence of CHD, and who were matched for the following cardiovascular risk factors: age at diagnosis of diabetes; sex; LDL-cholesterol (within $1 \mathrm{mmol} / 1$ ); HDL-cholesterol (within $0.3 \mathrm{mmol} / 1$ ); triglyceride (within $0.3 \mathrm{mmol} / \mathrm{l}$ ); and smoking status at enrolment (current/former/never). Lipid variables were measured at diagnosis. To enable assessment of whether any association of ACE genotype with MI could arise from an effect of ACE genotype on blood pressure, two groups of control subjects were selected: one matched for hypertensive status as determined by systolic blood pressure (mean of two readings taken 2 and 9 months after enrolment, matched
Data given as mean for normally distributed data and median (interquartile range) for non-normally distributed data, except where stated

${ }^{\mathrm{a}} p=0.04 ;{ }^{\mathrm{b}} p=0.012 ; \mathrm{c} p=0.01$

to within $10 \mathrm{~mm} \mathrm{Hg}$ ) and presence of antihypertensive treatment at diagnosis of diabetes in addition to the above variables; the other unmatched for hypertensive status. The clinical endpoint of MI was validated independently by two assessors [9]. Within the UKPDS, blood was drawn for genetic analysis commencing 1 January 1989 . By this time, 128 of the 5102 enrolled subjects had died. At the time of the current study, the total number of deaths in the UKPDS cohort was 499. Of the patients who died after enrolment $75 \%$ were therefore eligible for inclusion in the current study.

Genomic DNA was extracted using the Nucleon method (Scotlab Ltd. Paisley, UK) according to the manufacturer's instructions. DNA (500 ng) was amplified using the polymerase chain reaction, as described by Rigat et al. [10]. Thirty cycles of amplification were performed using a Perkin-Elmer/Cetus DNA Thermal Cycler (Norwalk, Conn., USA), with denaturation for $1 \mathrm{~min}$ at $94^{\circ} \mathrm{C}$, annealing for $1 \mathrm{~min}$ at $60^{\circ} \mathrm{C}$, and extension for $1 \mathrm{~min}$ at $72^{\circ} \mathrm{C}$. Each $25 \mu 1$ reaction contained PCR buffer ( $3 \mathrm{mmol} / 1$ magnesium chloride, $50 \mathrm{mmol} / 1$ potassium chloride, $10 \mathrm{mmol} / 1 \mathrm{Tris} \mathrm{HCl}\left(\mathrm{pH} 8.4\right.$ at $\left.20^{\circ} \mathrm{C}\right), 0.1 \%$ Triton $\mathrm{X}-100,0.2 \mathrm{mmol} / \mathrm{l} \mathrm{dNTPs}$ (Pharmacia, Uppsala, Sweden)), $1 \mathrm{U}$ Taq polymerase (Advanced Biotechnologies, London, UK), and $20 \mathrm{pmol}$ of each primer. Reaction products were electrophoresed on $2 \%$ agarose geis, stained with ethidium bromide, and viewed under ultraviolet light for allele identification.

In view of the concern about possible under-amplification of the I allele in ID heterozygotes [11] all the individuals with DD genotype using the above protocol were reamplified using an I-allele specific primer [12], with modification in reaction conditions such that denaturation took place at $93^{\circ} \mathrm{C}$, annealing at $65^{\circ} \mathrm{C}$, and extension at $72^{\circ} \mathrm{C}$. Where a DD sample amplified using the I-specific primer, it was coded ID.

\section{Statistical analysis}

Statistical analysis was performed using SAS (SAS Institute Inc, Cary, N.C., USA) on a VAX computer. Paired samples were compared for continuous variables using paired $t$-test, and for categorical data using McNemar's test statistic. Odds ratios are given with $95 \%$ confidence intervals, which were obtained using Mantel-Haenszel estimates. 
Table 2. ACE genotypes in cases of myocardial infarction and matched control subjects

\begin{tabular}{llllll}
\hline Genotype & \multicolumn{2}{l}{$\begin{array}{l}\text { Matched excluding } \\
\text { hypertensive status }\end{array}$} & & \multicolumn{2}{l}{$\begin{array}{l}\text { Matched including } \\
\text { hypertensive status }\end{array}$} \\
\cline { 2 - 3 } & Cases & $\begin{array}{c}\text { Control } \\
\text { subjects }\end{array}$ & & Cases & $\begin{array}{l}\text { Control } \\
\text { subjects }\end{array}$ \\
\hline DD $n(\%)$ & $46(28)$ & $36(22)$ & & $40(30)$ & $28(21)$ \\
ID & $80(49)$ & $88(54)$ & & $63(47)$ & $80(60)$ \\
II & $37(23)$ & $39(24)$ & & $31(23)$ & $26(19)$ \\
\hline
\end{tabular}

Data given as mean $(\mathrm{SD})$

\section{Results}

Table 1 shows the clinical and biological characteristics of the subjects. For the 173 cases selected, it was possible in 163 to match for risk factors excluding hypertensive status, and in 134 to match including hypertensive status. Comparison of the matched risk factors between cases and control subjects matched for hypertensive status showed no statistically significant differences. Glycated haemoglobin, which was an unmatched variable, was significantly higher in the case group $(p=0.04)$. Comparison between cases and control subjects unmatched for hypertensive status showed that diastolic blood pressure in cases was significantly higher than in the control subjects $(p=0.012)$ despite a significantly higher proportion of cases being on antihypertensive treatment ( $p=0.01$ ). Table 2 shows the frequencies of genotypes in case and control populations. The frequency of genotypes in the total population did not differ significantly from Hardy-Weinberg equilibrium.

Table 3 shows the comparison of ACE genotypes between cases and control subjects matched both including and excluding hypertensive status. A trend towards association of DD genotype with MI was observed when cases were compared with either control group. However, in neither comparison did the excess risk of MI conferred by DD reach statistical significance.

In accordance with the pre-existing data from Cambien et al. [4], that DD genotype is a risk factor in subjects classified as "low risk" on grounds of lipid levels and BMI, the population was stratified into a "high-risk" and a "low-risk" subgroup based on each of the matched cardiovascular risk factors in turn. For the continuous risk factors, the value used to stratify the population was the mean value in the case population. Accordingly, cases and control subjects with lower LDL-cholesterol, triglyceride, and age, and higher HDL-cholesterol than the mean of the case population were classified as "low-risk" individuals with respect to that risk factor. For the discontinuous matched risk factors, subjects who were not current smokers, who were not receiving antihypertensive medication, and who were female were classified as "low risk". The comparison of the matched pairs stratified for risk by LDL-cholesterol level in the 134 cases matched including hypertensive status is shown in Table 4. This analysis shows that the DD genotype was associated with MI in the low-LDL subgroup (odds ratio $3.67, p=0.002$ ). This analysis was re-

Table 3. Comparison of ACE genotypes in cases of myocardial infarction and matched control subjects

\begin{tabular}{lll}
\hline & $\begin{array}{l}\text { Matched excluding } \\
\text { hypertensive status }\end{array}$ & $\begin{array}{c}\text { Matched including } \\
\text { hypertensive status }\end{array}$ \\
\hline Both case and matched control subject of ID or II genotype & $88(54)$ & $75(56)$ \\
Both case and matched control subject of DD genotype & $7(4)$ & $9(7)$ \\
Case only of DD genotype; matched control ID or II & $39(24)$ & $31(23)$ \\
Control subject only of DD genotype; matched case ID or II & $29(18)$ & $19(14)$ \\
Excess of DD individuals in cases vs matched control subjects & $(39-29)=10$ & $(31-19)=12$ \\
Odds ratio for MI of DD vs ID + II & $1.35(0.83-2.17)$ & $1.63(0.93-2.87)$ \\
$p$ value & 0.23 & 0.09 \\
\hline
\end{tabular}

Odds ratio given with $95 \%$ confidence interval. Other values given as $n(\%)$

Table 4. Comparison of ACE genotypes in cases of myocardial infarction and control subjects matched including hypertensive status, stratified by LDL cholesterol

\begin{tabular}{lll}
\hline & LDL cholesterol <3.6 mmol/1 & LDL cholesterol > 3.6 mmol/1 \\
\hline Number of matched cases and control subjects & 67 & 67 \\
Both case and matched control subjects of ID or II genotype & $34(51)$ & $41(61)$ \\
Both case and matched control subject of DD genotype & $5(7)$ & $4(6)$ \\
Case only of DD genotype; matched control subject ID or II & $22(33)$ & $9(13)$ \\
Control subject only of DD genotype; matched case ID or II & $6(9)$ & $13(19)$ \\
Excess of DD individuals in cases vs matched controls & $(22-6)=16$ & $(9-13)=-4$ \\
Odds ratio for MI of DD vs ID + II & $3.67(1.58-8.51)$ & $0.69(0.3-1.61)$ \\
$p$ value & 0.002 & NS
\end{tabular}

Odds ratio given with $95 \%$ confidence interval. Other values given as $n(\%)$ 
Table 5. Odds ratios obtained on comparison of ACE genotypes in cases of myocardial infarction and control subjects matched in cluding hypertensive status after stratification based on matched cardiovascular risk factors

\begin{tabular}{llll}
\hline Risk factor & $\begin{array}{l}\text { Stratification applied } \\
\text { to define low-risk group }\end{array}$ & $\begin{array}{l}\text { Odds ratio for MI with DD } \\
\text { genotype in low-risk group }\end{array}$ & $\begin{array}{l}\text { Odds ratio for MI with DD } \\
\text { genotype in high-risk group }\end{array}$ \\
\hline LDL cholesterol & $<3.6 \mathrm{mmol} / 1$ & $3.67(1.58-8.51)^{\mathrm{a}}$ & $0.69(0.3-1.61)$ \\
Triglyceride & $<1.58 \mathrm{mmol} / 1$ & $3.14(1.4-7.04)^{\mathrm{a}}$ & $0.75(0.32-1.7)$ \\
HDL cholesterol & $>1.0 \mathrm{mmol} / \mathrm{l}$ & $1.4(0.62-3.14)$ & $1.9(0.85-4.1)$ \\
Age & $<58$ years & $0.92(0.42-2.02)$ & $3.17(1.33-7.55)$ \\
Smoking & Current non-smokers & $2.00(0.91-4.38)$ & $1.3(0.57-2.9)$ \\
Hypertension & No antihypertensive treatment & $1.5(0.68-3.32)$ & $1.78(0.8-3.9)$ \\
Male gender & Female gender & $1.75(0.87-3.52)$ & $1.43(0.55-3.73)$ \\
\hline
\end{tabular}

a Significant association of DD genotype with $\mathrm{MI}, p<0.05$ after Bonferroni correction

peated for each of the matched risk factors; a summary of the findings in the cases and control subjects matched including hypertensive status is presented in Table 5. After Bonferroni correction for 14 analyses, association of DD genotype with MI remained statistically significant in subjects with low LDL-cholesterol or low triglyceride $(p<0.05)$. When cases with both low LDL cholesterol and low triglyceride were compared with controls, the odds ratio of $\mathrm{MI}$ in cases with DD genotype was $9.0(2.7-30.0), p<0.001)$.

Repetition of the above analysis comparing cases with control subjects matched excluding hypertensive status yielded similar findings, but with lower odds ratios and degrees of statistical significance. The odds ratio for MI with DD genotype in cases with low LDL cholesterol was 2.44 (1.15-5.18), $p=0.02$, the odds ratio in cases with low triglyceride was $1.5(0.77-2.9), p=\mathrm{NS}$, and the odds ratio in cases with both low LDL cholesterol and low triglyceride was $2.5(1.0-6.2), p=0.05$.

As the study included patients with both fatal and non-fatal MI, these subgroups were additionally subjected to separate analysis. Of the 134 cases matched including hypertensive status, 38 (28\%) had suffered a fatal MI. When these 38 subjects were compared with their matched control subjects, the odds ratio for MI with DD genotype was 2.0 (0.70-2.77), $p=\mathrm{NS}$; when the remaining 96 cases matched including hypertensive status were compared with their matched control subjects, the odds ratio for MI with DD genotype was $1.5(0.77-2.94), p=\mathrm{NS}$. When the group of 38 deceased subjects was stratified by risk factors and compared with their matched control subjects, the odds ratio in cases with low LDL cholesterol was $2.7(0.7-9.6), p=\mathrm{NS}$, the odds ratio in cases with low triglyceride was $3.0(0.6-13.8), p=\mathrm{NS}$, and the odds ratio in cases with both low LDL cholesterol and low triglyceride was $6.0(0.94-38.5), p=\mathrm{NS}$.

\section{Discussion}

In this prospective case control study of NIDDM patients, we found that ACE-DD genotype was an im- portant risk factor for MI in subjects with low levels of blood lipids.

Analysis using two different control groups which were either matched or unmatched for blood pressure showed that the risk conferred by ACE-DD genotype was more apparent when the comparison was made between cases and control subjects which were matched for blood pressure. Cases in the group unmatched for blood pressure had significantly higher blood pressure than their matched control subjects. If ACE-DD genotype were acting in part by increasing the blood pressure, then any excess risk conferred by the genotype would be more apparent when comparison was made between cases and control subjects in the group unmatched for blood pressure. The effect of ACE-DD on risk of MI therefore could not arise by this mechanism.

The use of cases and control subjects drawn from the same study population and matched for known cardiovascular risk factors also permitted direct demonstration that the effect of ACE genotype in those with low levels of LDL and triglyceride was independent of all the other matched risk factors. Our results provide confirmation of a similar result obtained recently by Ruiz et al. [7], who used multiple logistic regression analyses to show that ACE genotype behaved as an independent risk factor for CHD in a cross-sectional case-control study of NIDDM subjects who were not matched for risk factors. Since risk factors were similarly distributed between our cases and control subjects, the risk conferred by the ACE genotype could be compared in groups stratified for each risk factor.

We have shown that the association of ACE-DD genotype with MI was present in cases with low LDL-cholesterol or triglyceride concentrations, and was particularly strong when both variables are low (the interaction between the two variables being multiplicative); this interaction of ACE-DD with favourable lipid levels is in accordance with the original report in the general population by Cambien et al. [4], who measured apo B concentration. This finding could have important implications. Multiple cardiovascular risk factors are generally held to act multipli- 
catively - an interaction of the type observed is highly unusual, and must be accommodated in any model of the pathophysiology. Alternatively, it has been proposed that the observed effect could arise from a survival bias for ascertainment $[5,6]$. According to this hypothesis "high-risk" DD individuals with high lipid levels may have a very adverse prognosis, and suffer fatal MI before being ascertained.

We think it unlikely that this explains our data. Firstly, we have reproduced the findings of Cambien et al. [4] in patients with NIDDM, a population which is at high risk of fatal MI even when lipid levels are relatively low. Secondly, the marked differences in odds ratio between "high-risk" and "lowrisk" subgroups observed when the population was stratified for lipid values was not observed when the population was stratified for other important risk factors affecting survival, such as smoking status, hypertension and age. Thirdly, although some bias could arise from selected entry to UKPDS [9], and from deaths in the period between entry to the study and the drawing of blood for DNA extraction, subsequent ascertainment of cases was prospective and included both fatal and non-fatal MIs. Moreover, separate analysis of cases who had died confirmed the trends observed in the entire population, although the small numbers involved resulted in these trends not reaching statistical significance.

Since the extent of linkage disequilibrium over this region of chromosome 17 is not known, it is not yet proven that the risk arises from ACE rather than a neighbouring gene. Nevertheless, ACE itself is an attractive candidate since ACE activity has been implicated in arterial wall myo-intimal proliferation in humans [13] and ACE inhibition can prevent atheroma formation in non-human primates [14]. Whereas high lipid levels may be directly pathogenic, genetic variability at the ACE locus could lead to increased pathogenicity of lower levels of blood lipids.

The clinical consequences of these findings could be significant. Among our NIDDM population, even those subjects with a favourable lipid profile have a substantially increased risk of MI when compared with the general population; in these subjects genotyping at the ACE locus may be an important part of overall cardiovascular risk stratification. Furthermore, evidence has been presented $[15,16]$ that in selected patients with left ventricular dysfunction after MI, ACE inhibition can not only improve mortality and progression to clinical heart failure, but also reduce the rate of recurrent MI. The possibility that the ACE genotype has effects on the response to $\mathrm{ACE}$ inhibition will require assessment in further studies.

Acknowledgements. BDK is supported by a Training Fellowship from the Medical Research Council of the United Kingdom
Hospitals participating in UKPDS: Radcliffe Infirmary, Oxford; Royal Infirmary, Aberdeen; General Hospital, Birmingham; St. George's Hospital, London; Hammersmith Hospital, London; City Hospital, Belfast; North Staffordshire Royal Infirmary, Stoke-on-Trent; Royal Victoria Hospital, Belfast; St. Helier Hospital, Carshalton; Whittington Hospital, London; Norfolk and Norwich Hospital; Lister Hospital, Stevenage; Ipswich Hospital; Ninewells Hospital, Dundee; Northampton Hospital; Torbay Hospital; Peterborough General Hospital; Scarborough Hospital; Derbyshire Royal Infirmary; Manchester Royal Infirmary; Hope Hospital, Salford; Leicester General Hospital; Royal Devon and Exeter Hospital, Exeter, UK.

\section{References}

1. Cambien F, Alhenc-Gelas F, Herbeth B et al. (1988) Familial resemblance of plasma angiotensin-converting enzyme level: the Nancy study. Am J Hum Genet 38: 228-234

2. Rigat B, Hubert C, Alhenc-Gelas F, Cambien F, Corvol P, Soubrier $F$ (1990) An insertion/deletion polymorphism in the angiotensin I-converting enzyme gene accounting for half the variance of serum enzyme levels. J Clin Invest 14: 1343-1346

3. Tiret L, Rigat B, Visvikis S et al. (1992) Evidence, from combined segregation and linkage analysis, that a variant of the angiotensin I-converting enzyme gene controls plasma ACE levels. Am J Hum Genet 51: 197-205

4. Cambien F, Poirier O, Lecerf L et al. (1992) Deletion polymorphism in the gene for angiotensin-converting enzyme is a potent risk factor for myocardial infarction. Nature 359: 641-644

5. Tiret L, Kee F, Poirier O et al. (1993) Deletion polymorphism in angiotensin-converting enzyme gene associated with parental history of myocardial infarction. Lancet 341: 991-992

6. Evans AE, Poirier O, Kee F et al. (1994) Polymorphisms of the angiotensin-converting enzyme gene in subjects who die from coronary heart disease. Quart J Med 87: 211-214

7. Ruiz J, Blanche H, Cohen N (1994) Insertion/deletion polymorphism of the angiotensin-converting enzyme gene is strongly associated with coronary heart disease in non-insulindependent diabetes mellitus. Proc Natl Acad Sci USA 91: 3662-3665

8. Bohn M, Berge KE, Bakken A, Erikssen J, Berg K (1993) Insertion/deletion polymorphism at the locus for angiotensinogen I-converting enzyme and myocardial infarction. Clin Genet 44: 292-297

9. UK Prospective Diabetes Study Group (1991) UK Prospective Diabetes Study (UKPDS). VIII. Study design, progress and performance. Diabetologia 34: 877-890

10. Rigat B, Hubert C, Corvol P, Soubrier F (1992) PCR detection of the insertion/deletion polymorphism of the human angiotensin converting enzyme gene (DCP1) (dipeptidyl carboxypeptidase 1). Nucleic Acids Res 20: 1433

11. Fogarty DG, Maxwell AP, Doherty CC, Hughes AE, Nevin NC (1994) ACE gene typing. Lancet 343: 851

12. Shanmugan V, Sell KW, Saha BK (1993) Mistyping ACE heterozygotes. PCR Methods Applications 3: 120-121

13. Powell JS, Clozel JP, Muller RKM et al. (1989) Inhibitors of angiotensin-converting enzyme prevent myointimal proliferation after vascular injury. Science 245: 186-188

14. Abert G, Ferrer P (1990) Effects of captopril on atherosclerosis in Cynomolgus monkeys. J Cardiovasc Pharmacol 15: S65-S72

15. Pfeffer MA, Braunwald E, Moye LA (1992) Effect of captopril on mortality and morbidity in patients with left ventricular dysfunction after myocardial infarction. Results of the survival and ventricular enlargement trial. N Engl J Med 327 (10): 669-677

16. The SOLVD Investigators (1992) Effect of enalapril on mortality and the development of heart failure in asymptomatic patients with reduced left ventricular ejection fractions. N Engl $J$ Med 327 (10): 685-691 